\title{
Numerical simulation of the compaction of crushable grains in 3D
}

\author{
David Cantor ${ }^{1,2, \star}$, Emilien Azéma ${ }^{1}$, Philippe Sornay ${ }^{2}$, and Farhang Radjai ${ }^{1,3}$ \\ ${ }^{1}$ Laboratoire de Mécanique et Génie Civil (LMGC), Université de Montpellier, CNRS, Montpellier, France \\ ${ }^{2}$ CEA, DEN, DEC, SFER, LCU, 13108, Saint Paul lez Durance, France \\ ${ }^{3}$ Multi-Scale Materials Science for Energy and Environment $<M S E>^{2}$, UMI 3466 CNRS-MIT Energy Initiative, Massachusetts \\ Institute of Technology, 77 Massachusetts Avenue, Cambrid02139, USA
}

\begin{abstract}
Grain fragmentation is simulated by means of a three-dimensional discrete element approach called bonded-cell method (BCM). In this method, grains and potential fragments may have any polyhedral shape and size, capturing the geometrical complexity of brittle grain failure. As an application of this method, we present the uniaxial compaction of samples composed of several grains and we analyse the load-density relations, the grain size evolution, and the failure mechanism within the grains. This numerical approach permitted us to analyse the effect of the grains internal strength on the macroscopic compaction behaviour and to study the evolution of the grain size distribution towards a power-law distribution as several experiments have shown in literature. Finally, we present a brief micro-mechanical analysis on the failure modes within the grains, letting us know the kind of stresses that prompts grain fragmentation.
\end{abstract}

\section{Introduction}

Fragmentation of brittle grains has major effects on the compressive and shear strength of granular materials [1, 2], packing fraction [10], the yielding surface [11], grain size distribution [8], etc. Most of these observations have been conducted or analysed through experimental or empirical approaches and, together with the concepts of fracture mechanics, they provide a remarkable insight into the behaviour of breakable grains.

Nevertheless, the simulation of grain fragmentation remains still a challenge. Several models have been proposed to deal with fragmentation in the framework of continuum or discrete element methods (some examples can be found in Ref. [4] and Refs. [5, 15], respectively). These numerical methods contain, the most of the times, two main ingredients: the failure criterion and the geometry evolution. They define the stress level to split a grain apart and the shape of fragments. Due to the rapid evolution of grain geometry and grain size distribution during any fragmentation event, the computational cost of these models may be very important. Then, very simplified models have been, up to now, the way to tackle this phenomenon. For instance, the clustering of discs or spheres, in 2D and 3D simulations respectively, are the most common techniques to deal with grain failure. However, the shape complexity of grains and fragments is needed to properly map the macroscopic response from the grain scale interactions. For this reason, and inspired by the two-dimensional strategy of Nguyen et al. [12], we have introduced a three-dimensional discrete element approach

\footnotetext{
^e-mail: david.cantor@umontpellier.fr
}

to simulate fragmentation [3]. In this model, each grain is composed of a set of potential fragments, also known as cells, bonded with a couple of mechanical constrains or bonding strengths. When the intercell forces reach such strengths, the grains are allowed to fail in arbitrary shapes as their interactions and external loads may determine it.

This article is organized as follows: In Section 2, we briefly present the numerical model for grain fragmentation and the numerical procedure to perform uniaxial compressions on assemblies of grains. In Section 3, we present some results on the behaviour of such assemblies concerning the load-density relations, the grain size evolution, and the failure mechanism within the grains. Finally, we discuss the current results and potential perspectives of this work.

\section{Numerical experiments}

The discrete element approach that we use to simulate grain fragmentation is known as bonded-cell method (BCM) [3, 12]. As an application of the BCM, we performed uniaxial compression tests on assemblies of crushable grains. This kind of test was chosen given its simplicity and its well-known macroscopic response. In the two following Subsections, we briefly present the BCM$3 \mathrm{D}$ and the numerical procedures to perform the compression tests.

\subsection{Bonded-Cell Method in 3D}

This numerical method is based on the subdivision of grains in a set of potential fragments or cells. These frag- 


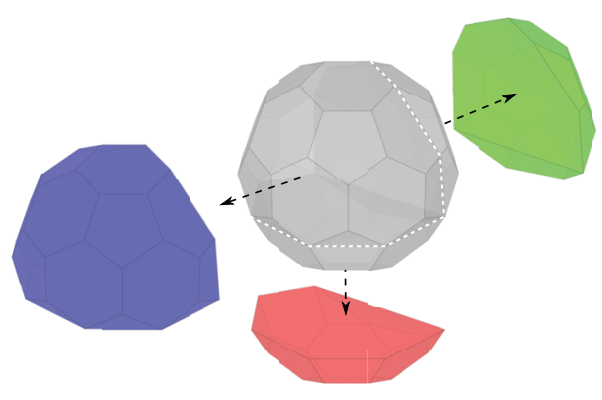

Figure 1. Exploded view of a convex grain composed of 3 cells.

ments are obtained after a Voronoi tessellation of the original grain. By changing the number of generators in the tessellation, it is possible to control the number of potential fragments $\left(n_{\mathrm{cl}}\right)$. Also, it is possible to modify the geometry of fragments by controlling the dislocation of the centroid of the cells with respect to the position of its corresponding generator [6]. A weighting of such dislocation for all the cells within a grain let us define a geometrical degree of disorder $(\lambda)$ that may vary between 0 and 1 . When $\lambda$ tends to 0 , the cells have almost-regular shapes and they are well distributed within the grain. As $\lambda$ increases, the cells may have elongated shapes and varying sizes. Figure 1 shows an example of a grain with 3 cells highlighting the way it is composed in an exploded view. The intercell bond is determined by two parameters: a tensile strength $\left(C_{t}\right)$ acting in the normal contact direction, and a shear strength $\left(C_{s}\right)$ present in the tangential contact direction. Once the forces between two cells reach either $C_{t} \cdot S$ or $C_{s} \cdot S$, with $S$ equals to the contact area, the bond is permanently lost (i.e., a fissure is created along the intercell contact surface). These broken surfaces will behave, in case of a new interaction with other cells, as dry surfaces with a coefficient of friction $\mu$. It has been shown that parameters $n_{\mathrm{cl}}$ and $\lambda$ can modify the strength of individual grains under diametral compression. However, it is the intercell strength that mainly controls the compressive behaviour of individual grains [3]. For the following numerical experiments, and for the sake of simplicity, we fixed $n_{\mathrm{cl}}=10, \lambda=0.25$ and $\mu=0.4$.

We used the discrete element method known as contact dynamics for our simulations, in which the equation of motion is integrated in an implicit time stepping scheme and the cells are considered as rigid bodies [13]. In effect, overlapping between bodies do not represent the contact deformations and only are taken into account during the contact detection. In addition, the cells velocities and contact forces are computed by means of a Gauss-Seidel iterative algorithm as function of the external loads and the rigid bodies interactions.

\subsection{Compression tests}

Assemblies of crushable grains were built by depositing polyhedral grains into cubic boxes. The assemblies were composed of 1000 grains, and each grain had 10 cells. For each sample, the intercell bonding strength varied between

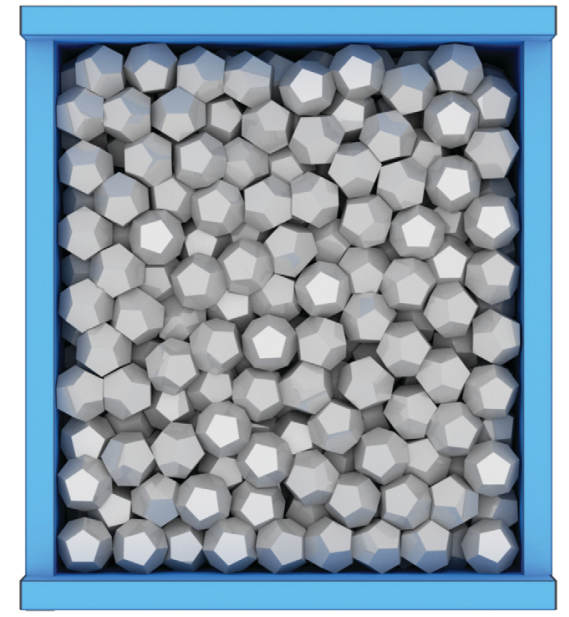

(a)

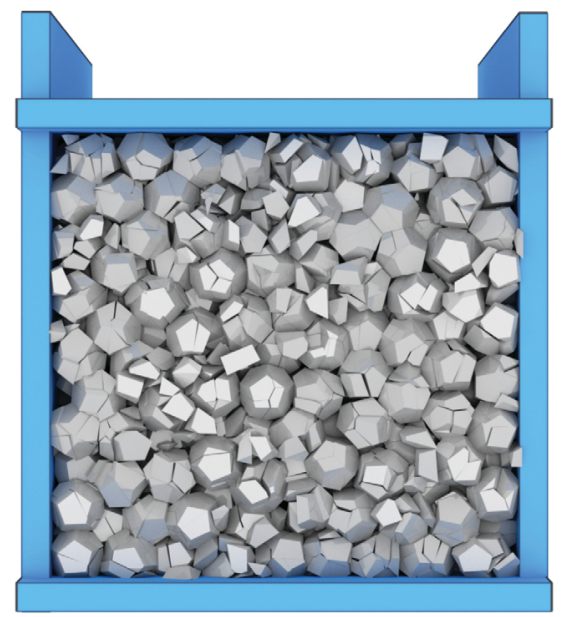

(b)

Figure 2. Front view of a sample composed of 1000 grains as the applied vertical stress increases from (a) $\sigma_{h}=0$, to (b) $\sigma_{h}=2 C_{t}$. The frontal wall was removed for the visualization.

0.1 and $10 \mathrm{MPa}$. Then, with the aim of having dense samples, isotropic compressions were carried out keeping in mind that the imposed stresses were light enough to prevent fragmentation during this test stage. Finally, uniaxial compression tests were undertaken on each assembly.

Figure 2 shows screenshots of a sample as the vertical load $\sigma_{h}$ is increased. In this figure, it is possible to identify grains that are being fragmented as well as others that remain intact throughout the compaction. In general, grains transmitting an important part of the vertical load are more susceptible to break while grains outside such force networks are barely affected.

\section{Results}

In this section, we present some results concerning the relation between the external load and the sample density, the grain size evolution, and the fragmentation mode within the grains. The first two parameters mentioned above are measures at the sample scale, and they are usu- 


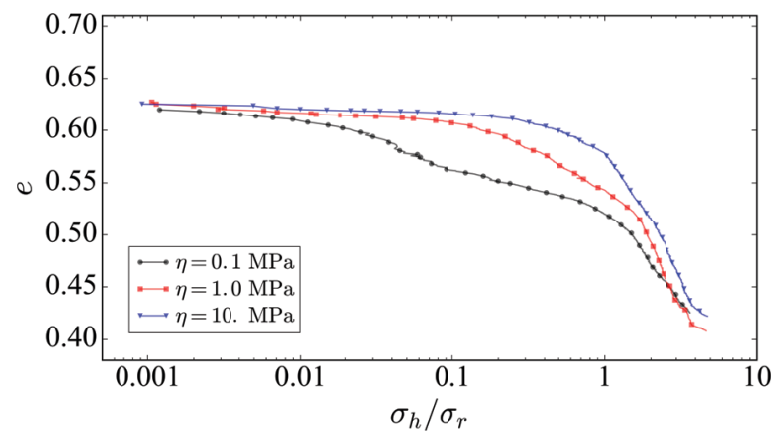

Figure 3. Evolution of the void ratio as function of the normalized vertical load for samples presenting different intercell bonding strength $\eta$.

ally found in experimental testing. The fragmentation mode is, instead, a measure at the grain scale and it describes the type of stresses that are causing the loss of bonds between cells.

\subsection{Load vs density}

In our experiments, the sample density is analysed through the void ratio $(e)$ that is the parameter relating the volume of voids in the sample $\left(V_{v}\right)$ and the volume of grains $\left(V_{s}\right)$ $\left(e=V_{v} / V_{s}\right)$. As the vertical stress increases, the volume of the container is reduced and the void ratio should decrease as well. Figure 3 shows the evolution of $e$ as the ratio $\sigma_{h} / \sigma_{r}$ increases (i.e., the imposed stress normalized by a stress of reference $\left.\sigma_{r}=1 \mathrm{MPa}\right)$. For the sake of simplicity, and given that $C_{t}$ and $C_{n}$ were increased simultaneously in different tests, let us simplify the notation as follows: $C_{t}=C_{n}=\eta$. Two different regimes during the compaction can be differentiated. The first one corresponds to that where the volume is slowly reduced as the load starts to increase. During the second stage, the void ratio decreases more rapidly. The transition between these two regimes is affected in particular by the intercell strength. Even if this effect seems to be relatively small, when compared with the general behaviour during the compaction, it evidences the increase of energy per unit of volume that is being stored in the intact bonds. We could also observe that fragmentation occurs since the beginning of the loading and it does not stop till the end of the second regime. At that point, not all the bonds are broken but the dense arrangement of grains prevents more fragmentation events.

\subsection{Grain size evolution}

Figure 4 shows the evolution of the grain size distribution (i.e., the cumulative probability $h$ of having a grain of diameter $d$ ), for the sample with $\eta=1 \mathrm{MPa}$, as function of the measured vertical strain. As it can be observed, the sample starts as a mono-disperse assembly of grains. Different grain size distributions are presented for 4 values of vertical strain $\left(\varepsilon_{h}\right)$ during the compaction. As indicated in the Figure, the dashed line corresponds to a power-law

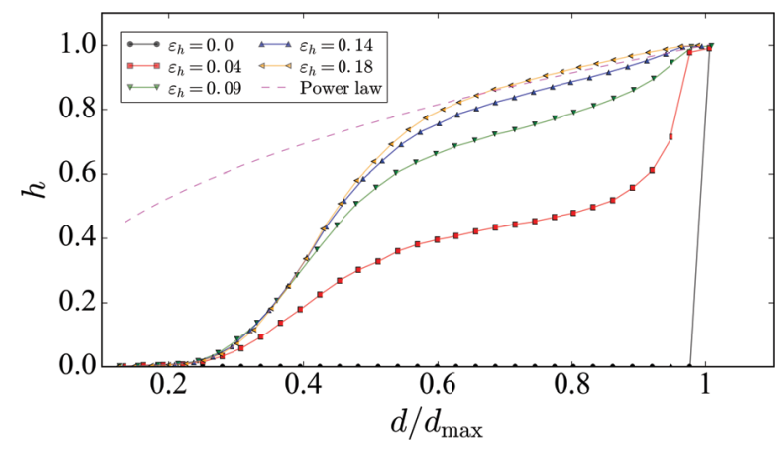

Figure 4. Evolution of the grain size distribution for 5 different instants during the compaction. The dashed line corresponds to the ultimate grain size distribution repetitively found in literature. Such distribution has a power-law shape of the form $h^{*}(d)=\left(d / d_{\max }\right)^{3-\alpha}$, with $\alpha=2.6$.

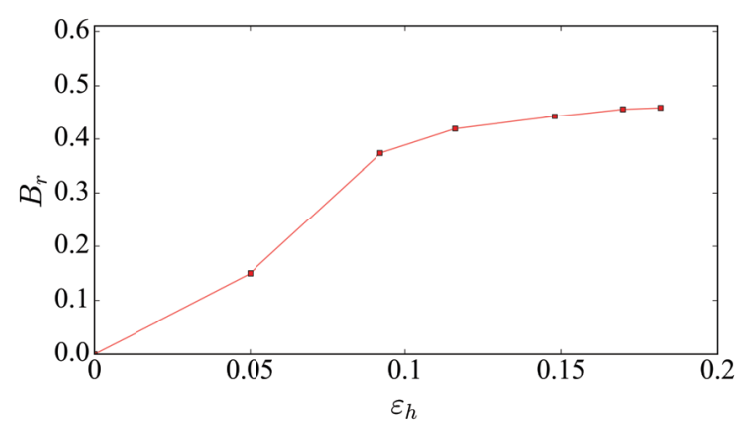

Figure 5. Evolution of the fragmentation index as function of the vertical strain for one of the samples tested.

distribution, being $d_{\max }$ the maximum particle size in the sample, and $\alpha=2.6$. This exponent $\alpha$ has been repetitively found in experiments where granular samples undergo large shear deformations [14]. Regardless of the fact that in our model fragmentation events are constrained by the size of the smallest cell and the available space between grains, the grain size distribution tends partially to such power distribution. These grain size distributions may be compared by means of a fragmentation index $B_{r}$ $[7,8]$, that can be calculated as follows:

$$
B_{r}=\frac{\int_{d_{\min }}^{d_{\max }}\left(h_{0}(d)-h(d)\right) \mathrm{d} d}{\int_{d_{\min }}^{d_{\max }}\left(h_{0}(d)-h^{*}(d)\right) \mathrm{d} d},
$$

where $h_{0}$ is the grain size distribution at the beginning of the simulation. Figure 5 shows the evolution of $B_{r}$ as function of the vertical strain. It indicates that, in the case of uni-axial compression tests, the fragmentation reaches at most $50 \%$ of the potential fragmentation $h^{*}(d)$ for this material . Nevertheless, we can observe the grain size distribution will hardly evolve further than such distribution shown in Fig. 4 for $\varepsilon_{h}=0.18$. Then, the potential grain size distribution should be indeed revised to have a better evaluation of grain fragmentation during uni-axial compression tests. 


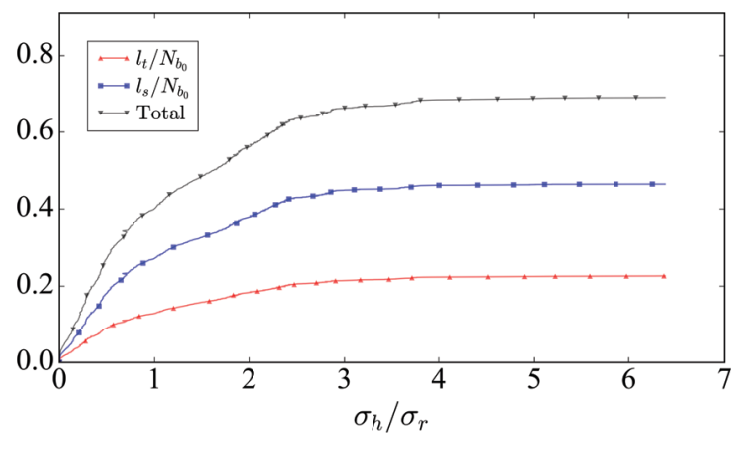

Figure 6. Intercell failure mode classification in contacts lost by tensile $\left(l_{t}\right)$ or shear $\left(l_{s}\right)$ stresses normalized by the initial number of bonds $N_{b_{0}}$.

\subsection{Fragmentation mode}

By means of our discrete element approach, we were able to identify the bonds that are lost by tensile or shear stresses. Figure 6 shows the number of bonds lost either in traction $l_{t}$ or shear $l_{s}$ modes normalized by the initial number of bonds $\left(N_{b_{0}}\right)$, as function of the external load $\sigma_{h}$, for the samples with $\eta=1 \mathrm{MPa}$. In this figure, we can remark that most of the bonds are lost by shear stresses. We could determine that tensile failures are rare as the load increases, and they are usually consequence of previous shear failures that push apart other cells by induced indirect traction. Also, the increase of grain coordination (average number of contacts per grains), reduces the probability of having cells that fail in the tensile mode.

\section{Discussion and perspectives}

We have implemented a three-dimensional model to grain fragmentation known as bonded-cell method (BCM), based on the bonding of a grain potential fragments or cells. With this approach, the fragmentation events are function of the external loads and interaction between the grains, and it allows fragments to evolve into diverse shapes and sizes. We analysed the behaviour of assemblies of particles under uni-axial compression. In particular, we studied the load-density relations, the evolution of the grain size distribution, and the type of stresses within the grains that are causing failure. Our results are in very good agreement with experimental testing performed in soil and rock mechanics. For instance, this work presented the evolution of density as the vertical load increases. It let us observe how the grain size of fragments evolves towards a well-known power-law distribution, as well as we could identify the roll of the intercell strength on the energy storage within intact the bonds. Finally, we showed that shear failure is the principal mechanism of fragmentation in uniaxial compression tests. This discrete element approach permits to clearly map the macroscopic behaviour from the grain and contacts scale, making our 3D bonded-cell method an outstanding framework to study crushable materials. Future works with the BCM-3D will include the study of the shape variability of fragments, the evolution of number of contacts per fragment, and analysis on the consumption of energy as fragmentation occur.

\section{Acknowledgement}

This work was financially supported by the French Alternative Energies and Atomic Energy Commission (CEA).

\section{References}

[1] Aleai, E., Mahboudi, A.: Discrete model for simulating shear strength and deformation behaviour of rockfill material, considering the particle breakage phenomenon. Granular Matter 14(6), 707-717, (2012)

[2] Arslan, H., Baykal, G., Sture, S.: Analysis of the influence of crushing on the behavior of granular materials under shear. Granular Matter 11(2), 87-97, (2009)

[3] Cantor, D., Azéma, E., Sornay, P., Radjai, F.: Threedimensional bonded-cell model for grain fragmentation. Computational Particle Mechanics, 1-10, (2016)

[4] Cecconi, M., DeSimone, A., Tamagnini, C., M.B. Viggiani, G.: A constitutive model for granular materials with grain crushing and its application to a pyroclastic soil. Int J Numer Anal Met 26(15), 1531-1560, (2002)

[5] Cheng, Y. P., Bolton, M. D., Nakata, Y.: Discrete element simulation of crushable soil. Géotechnique 53(7), 633-641, (2003)

[6] Du, Q., Faber, V., Gunzburger, M.: Centroidal Voronoi Tessellations: Applications and Algorithms. SIAM Review 41(4), 637-676, (1999)

[7] Einav, Itai.: Breakage mechanics - Part I: Theory. J Mechan Phys Solids 55(6), 1274-1297, (2007)

[8] Hardin, B. O.: Crushing of Soil Particles. Journal of Geotechnical Engineering 111(10), 1177-1192, (1985)

[9] Hégron, L., Sornay, P., Favretto-Cristini, N. Compaction of a bed of fragmentable UO2 particles and associated acoustic emission. IEEE Transactions on $\mathrm{Nu}-$ clear Science, 61(4), 2175-2181, (2014)

[10] Kong, C. M., Lannutti, J. J.: Localized densification during the compaction of alumina granules: The Stage I-II transition. Journal of the American Ceramic Society $\mathbf{8 3}, 685-690,(2000)$

[11] Miura, N., Murata, H., Yasufuku, N.: Stress-strain characteristics of sand in a particle-crushing region. Soils and Foundations 24(1), 77-89, (1984)

[12] Nguyen, D-H., Azéma E., Sornay, P., Radjai, F.: Bonded-cell model for particle fracture. Physical Review E 91(2), 022203, (2015)

[13] Radjai, F., Richefeu, V.: Contact dynamics as a nonsmooth discrete element method. Mechanics of Materials, 41(6), 715-728, (2009)

[14] Sammis, C., King, G., Biegel, R.: The kinematics of gouge deformation. Pure and Applied Geophysics, 125(5), 777-812, (1987)

[15] Tsoungui, O., Vallet, D., Charmet, J. C.: Numerical model of crushing of grains inside two-dimensional granular materials. Powder Technology 105 (1-3), 190198, (1999) 